\title{
PRODUCTS OF IDEMPOTENT ENDOMORPHISMS OF AN INDEPENDENCE ALGEBRA OF FINITE RANK
}

\author{
by JOHN FOUNTAIN and ANDREW LEWIN
}

(Received 8th October 1990)

\begin{abstract}
Products of idempotents are investigated in the endomorphism monoid of an algebra belonging to a class of algebras which includes finite sets and finite dimensional vector spaces as special cases. It is shown that every endomorphism which is not an automorphism is a product of idempotent endomorphisms. This provides a common generalisation of earlier results of Howie and Erdos for the cases when the algebra is a set or vector space respectively.
\end{abstract}

1991 Mathematics subject classification (1985 Revision): 20M20

\section{Introduction}

For a mathematical structure $M$ we denote the set of endomorphisms of $M$ by End $(M)$ and the set of automorphisms of $M$ by Aut $(M)$. Under composition of mappings, $\operatorname{End}(M)$ is a monoid and $\operatorname{Aut}(M)$ is a subgroup of this monoid. We let $E$ denote the set of non-identity idempotents of $\operatorname{End}(M)$. Over the last twenty-five years considerable effort has been devoted to describing the subsemigroup $\langle E\rangle$ generated by $E$. The first results were obtained by Howie in [7] where a set-theoretic description of $\langle E\rangle$ is given when $M$ is simply a set and $\operatorname{End}(M)$ is the full transformation semigroup on $M$. For the case when $M$ is a finite set, the result is:

$$
\langle E\rangle=\operatorname{End}(M) \backslash \operatorname{Aut}(M)
$$

When $M$ is a finite dimensional vector space, J. A. Erdos [3] proved the same result. An alternative proof was given later by Dawlings [1].

The object of the present paper is to prove the result for a class of algebras, called independence algebras, of which sets and vector spaces are specific instances. We thus obtain a common generalisation of the theorems of Howie and Erdos.

In [7], Howie also described $\langle E\rangle$ when $M$ is an infinite set and the analogous result for an infinite dimensional vector space $M$ was found by Reynolds and Sullivan [11]. A common generalisation of these theorems for a special class of independence algebras is the subject of a subsequent paper.

Independence algebras were defined by Gould in [4] where she describes the basic semigroup structure of the endomorphism monoids of such algebras. In fact, independence algebras are precisely the $v^{*}$-algebras introduced by Narkiewicz [10] and 
described in [5]. However, we follow Gould's formulation of the concept as this is designed to facilitate the study of the endomorphism monoid of the algebra. We give the appropriate definitions and terminology in Section 1 and follow this with a summary of some of Gould's results on the endomorphism monoids of independence algebras. The second section of the paper is devoted to proving the main theorem.

\section{Preliminaries}

For standard concepts of semigroup theory see, for example, [8]. For universal algebra terminology and notation we follow [9] with the exception that we denote the subalgebra generated by a subset $X$ of an algebra $A$ by $\langle X\rangle$. If the algebra $A$ contains constants, that is, values of nullary operations, then we denote the subalgebra generated by the constants by $C o n$ and make the convention that $\langle\varnothing\rangle=C o n$. A subset $X$ of an algebra $A$ is said to be independent if $X=\varnothing$ or for every element $x$ of $X$ we have $x \notin\langle X \backslash\{x\}\rangle ; X$ is dependent if it is not independent. Clearly, every singleton set consisting of a non-constant element of $A$ is independent.

A standard Zorn's lemma argument shows that, given subsets $X_{0}, X$ of $A$ with $X_{0}$ independent and contained in $X$, there is an independent subset $Y$ of $A$ with $X_{0} \subseteq Y \subseteq X$ such that $Y$ is maximal among independent sets contained in $X$. The following result is from [9, p. 50, Exercise 6].

Proposition 1.1. For an algebra $A$, the following conditions are equivalent:

(1) For every subset $X$ of $A$ and all elements $u$, $v$, of $A$, if $u \in\langle X \cup\{v\}\rangle$ and $u \notin\langle X\rangle$, then $v \in\langle X \cup\{u\}\rangle$.

(2) For every subset $X$ of $A$ and every element $u$ of $A$, if $X$ is independent and $u \notin\langle X\rangle$, then $X \cup\{u\}$ is independent.

(3) For every subset $X$ of $A$, if $Y$ is a maximal independent subset of $X$, then $\langle X\rangle=\langle Y\rangle$.

(4) For subsets $X, Y$ of $A$ with $Y \subseteq X$, if $Y$ is independent, then there is an independent set $Z$ with $Y \subseteq Z \subseteq X$ and $\langle Z\rangle=\langle X\rangle$.

An algebra $A$ is said to have the exchange property or to satisfy [EP] if it satisfies the equivalent conditions of Proposition 1.1. A basis for $A$ is a subset of $A$ which generates $A$ and is independent. It is clear from Proposition 1.1 that any algebra with the exchange property has a basis. Furthermore, for such an algebra $A$, bases may be characterised as minimal generating sets or maximal independent sets, and all bases for $A$ have the same cardinality. This cardinal is called the $\operatorname{rank}$ of $A$ and is written as $\operatorname{rank} A$.

We emphasise that (4) of Proposition 1.1 tells us that any independent subset of $A$ can be extended to a basis for $A$. We also remark that it is clear that if $A$ satisfies [EP], then so does any subalgebra of $A$.

We now define an independence algebra to be an algebra $A$ which satisfies [EP] and also satisfies: 
[F] For any basis $X$ of $A$ and any function $\alpha: X \rightarrow A$, there is an endomorphism $\bar{\alpha}$ of $A$ such that $\left.\bar{\alpha}\right|_{X}=\alpha$.

Condition $[\mathrm{F}]$ is equivalent to asserting that $A$ is free in the variety it generates and that any basis is a set of free generators. We note that if $A$ is an independence algebra and $Y$ is an independent subset of $A$ and $\alpha: Y \rightarrow A$ is any function, then there is a homomorphism $\bar{\alpha}:\langle Y\rangle \rightarrow A$ which extends $\alpha$. This follows from [F] since, by the exchange property, $Y$ can be extended to a basis $X$ for $A$ and then any extension of $\alpha$ to $X$ gives rise to an endomorphism of $A$ which restricts to give the required homomorphism.

It is easily seen that this homomorphism is uniquely determined by $\alpha$. Thus if the endomorphisms $\theta$ and $\psi$ agree on a basis for $A$, then $\theta=\psi$.

Familiar examples of independence algebras are sets (where all subsets are independent), vector spaces (where the independent subsets are the linearly independent subsets) and for any group $G$, free $G$-sets (where the independent sets are subsets of free generating sets).

Let $A$ be an independence algebra. The rank of an endomorphism $\alpha$ of $A$ is defined to be rank of the subalgebra $\operatorname{Im} \alpha$. We quote the following lemma from [4].

Lemma 1.2. Let $A$ be an independence algebra. If $\alpha, \beta \in \operatorname{End}(A)$, then rank $\alpha \beta \leqq$ min $\{\operatorname{rank} \alpha, \operatorname{rank} \beta\}$.

As a consequence of this lemma, for each cardinal $\kappa$ with $\kappa \leqq$ rank $A$, the set

$$
T_{\kappa}=\{\alpha \in \operatorname{End}(A): \operatorname{rank} \alpha \leqq \kappa\}
$$

is an ideal of $\operatorname{End}(A)$. When $A$ has finite rank $n$ we also use the notation $K(n, r)$ to denote $T$, for $r \leqq n$.

The following description of Green's relations on the endomorphism monoid of an independence algebra is taken from [4].

Proposition 1.3. Let $A$ be an independence algebra. Then for $\alpha, \beta \in \operatorname{End}(A)$,

(1) $\alpha \mathscr{L} \beta$ if and only if $\operatorname{Im} \alpha=\operatorname{Im} \beta$,

(2) $\alpha \mathscr{R} \beta$ if and only if $\operatorname{Ker} \alpha=\operatorname{Ker} \beta$,

(3) $\alpha \mathscr{D} \beta$ if and only if rank $\alpha=\operatorname{rank} \beta$,

(4) $\mathscr{D}=\mathscr{J}$.

It follows from this proposition that the principal ideals of $\operatorname{End}(A)$ are precisely the ideals $T_{\kappa}$ for $\kappa \leqq \operatorname{rank} A$. Other ideals exist only when rank $A$ is infinite and when this is the case the remaining ideals are the sets

$$
I_{\kappa}=\{\alpha \in \operatorname{End}(A): \operatorname{rank} \alpha<\kappa\}=\bigcup\left\{T_{\lambda}: \lambda<\kappa\right\}
$$

for limit cardinals $\kappa$. 
If $\operatorname{Con} \neq \varnothing$, then $T_{0} \neq \varnothing$ and $T_{0}$ is a principal factor of $\operatorname{End}(A)$. Otherwise, $T_{0}=\varnothing$ and $T_{1}$ is a principal factor. The remaining principal factors are the Rees quotients $T_{\kappa}+/ T_{\kappa}$ where $\kappa^{+}$is the successor of $\kappa$, and $T_{\kappa} / I_{\kappa}$ for limit cardinals $\kappa$.

For each positive integer $n$ we denote the principal factor $T_{n+1} / T_{n}$ by $P_{n+1}$ and the $\mathscr{D}$-class of endomorphisms of rank $n$ by $D_{n}$. Then $P_{n+1}=D_{n+1} \cup\{0\}$ with the product of two members of $D_{n+1}$ being zero if and only if the product in $\operatorname{End}(A)$ is not in $D_{n+1}$. If Con $\neq \varnothing$, then $P_{1}=T_{1} / T_{0}$ and $P_{0}=T_{0}$; otherwise, $P_{1}=T_{1}$.

We require two more results from [4].

Proposition 1.4. For each positive integer $n$, the principal factor $P_{n}$ is completely 0 -simple (or completely simple if $n=1$ and $P_{1}=T_{1}$ ).

In [4] Gould gives an explicit representation of $P_{n}$ as a Rees matrix semigroup.

Lemma 1.5. Let $\alpha$ be an endomorphism of an independence algebra $A$. If $\left\{x_{1}, \ldots, x_{k}\right\}$ is $a$ basis for $\operatorname{Im} \alpha$ and if $y_{1}, \ldots, y_{\kappa} \in A$ are such that $y_{i} \alpha=x_{i}$ for $i=1, \ldots, k$, then $\left\{y_{1}, \ldots, y_{k}\right\}$ is independent.

\section{The main theorem}

Let $A$ be an independence algebra and $E$ be the set of idempotents in $\operatorname{End}(A) \backslash \operatorname{Aut}(A)$. We devote this section to the proof of the following theorem.

Theorem 2.1. If rank $A=n$ is finite, then

$$
\langle E\rangle=\left\langle E_{1}\right\rangle=\operatorname{End}(A) \backslash \operatorname{Aut}(A)
$$

where $E_{1}$ is the set of idempotents of rank $n-1$ in $\operatorname{End}(A)$.

The strategy of the proof is inspired by an outline of a proof given in [2] for the case when $A$ is a vector space. Let

$$
S=K(n, n-1)=\operatorname{End}(A) \backslash \operatorname{Aut}(A) .
$$

We show first that $D_{n-1}$ generates $S$; in fact, we show that $D_{n}$ generates $K(n, r)$. Next we consider a group $\mathscr{H}$-class $H$ contained in $D_{n-1}$. We show that any $\mathscr{H}$-class in the same $\mathscr{R}$-class as $H$ or in the same $\mathscr{L}$-class as $H$ contains an element which is a product of idempotents. It then follows from Green's Lemma that $P_{n-1}$ is generated by $H \cup E_{1}$. Finally, this allows us to show that $P_{n-1}$ is generated by $E_{1}$ and the theorem follows.

For the remainder of the paper, $A$ denotes an independence algebra of rank $n$. If $n=1$, then either $\operatorname{Con}=\varnothing$ and $K(1,0)=\varnothing$ or $A$ contains constant and $K(1,0)$ consists of all endomorphisms $\alpha$ with Im $\alpha=$ Con. Since all such endomorphisms are idempotent, it is certainly true that $K(1,0)$ is generated by idempotents. We may therefore assume henceforth that $n \geqq 2$. 
Lemma 2.2. Let $\alpha \in D_{r-1}$ where $r<n$. Then there are endomorphisms $\beta, \gamma$ in $D_{r}$ such that $\alpha=\beta \gamma$.

Proof. If $A$ contains constants, then $r$ can be 1 . In this case, let $\left\{x_{1}, \ldots, x_{n}\right\}$ be a basis for $A$ and define $\beta \in \operatorname{End}(A)$ by specifying $x_{1} \beta=x_{1}, x_{i} \beta=x_{i} \alpha$ for $i=2, \ldots, n$. Then $\operatorname{Im} \beta=\left\langle x_{1}\right\rangle$ so that $\beta \in D_{1}$. Now define $\gamma \in \operatorname{End}(A)$ by putting $x_{1} \gamma=x_{1} \alpha, x_{i} \gamma=x_{1}$ for $i=2, \ldots, n$. Since $2 \leqq n$, it is clear that $\gamma$ has rank 1 . Further, it is equally clear that $x_{i} \beta \gamma=x_{i} \alpha$ for $i=1, \ldots, n$ so that $\beta \gamma=\alpha$ as required.

Now suppose that $1<r$. Then there is a basis $\left\{x_{1}, \ldots, x_{r-1}\right\}$ for $\operatorname{Im} \alpha$; this is contained in a basis $\left\{x_{1}, \ldots, x_{n}\right\}$ for $A$. Choose $y_{1}, \ldots, y_{r-1}$ in $A$ with $y_{i} \alpha=x_{i}$ for $i=1, \ldots, r-1$; then, by Lemma $1.5,\left\{y_{1}, \ldots, y_{r-1}\right\}$ is independent and so there is a basis $\left\{y_{1}, \ldots, y_{r-1}\right.$, $\left.y_{r}, \ldots, y_{n}\right\}$ for $A$. For $i=r, \ldots, n$ we have $y_{i} \alpha \in\left\langle x_{1}, \ldots, x_{r-1}\right\rangle$.

Define endomorphisms $\beta$ and $\gamma$ as follows:

$$
y_{i} \beta= \begin{cases}x_{i} & \text { for } 1 \leqq i \leqq r \\ y_{i} \alpha & \text { for } r<i \leqq r\end{cases}
$$

and

$$
x_{i} \gamma= \begin{cases}x_{i} & \text { for } 1 \leqq i \leqq r-1 \\ y_{i} \alpha & \text { for } i=r \\ x_{r} & \text { for } r<i \leqq n\end{cases}
$$

It is readily seen that $\alpha=\beta \gamma$ and that

$$
\operatorname{Im} \beta=\operatorname{Im} \gamma=\left\langle x_{1}, \ldots, x_{r}\right\rangle
$$

so that $\beta$ and $\gamma$ both have rank $r$.

As a consequence of this lemma, a set of elements of rank $r$ generates $P_{r}$ if and only if it generates $K(n, r)$.

Lemma 2.3. If $\phi, \gamma$ are idempotents in $D_{n-1}$, then there is an idempotent $\varepsilon$ in $D_{n-1}$ such that $\phi \varepsilon \gamma \in D_{n-1}$.

Proof. Let $\left\{x_{1}, \ldots, x_{n-1}\right\}$ be a basis for $\operatorname{Im} \phi$; then $x_{i} \phi=x_{i}$ for $i=1, \ldots, n-1$ since $\phi$ is idempotent. Let $x_{n} \in A$ be such that $\left\{x_{1}, \ldots, x_{n}\right\}$ is a basis for $A$. Then

$$
\operatorname{Im} \gamma=\left\langle x_{1} \gamma, \ldots, x_{n} \gamma\right\rangle
$$

and since $\gamma$ has rank $n-1$, there is an independent subset of $\left\{x_{1} \gamma, \ldots, x_{n} \gamma\right\}$ of cardinality $n-1$. 
If $\left\{x_{1} \gamma, \ldots, x_{n-1} \gamma\right\}$ is independent, then since $x_{i} \phi \gamma=x_{i} \gamma$ for $i=1, \ldots, n-1$, it follows that $\phi \gamma$ has rank $n-1$ and so taking $\varepsilon=\phi$ we have $\phi \varepsilon \gamma, \varepsilon \in D_{n-1}$.

Now suppose that $\left\{x_{1} \gamma, \ldots, x_{n-1} \gamma\right\}$ is dependent; then without loss of generality we may suppose that $\left\{x_{2} \gamma, \ldots, x_{n} \gamma\right\}$ is independent.

If $x_{n} \gamma \in\left\langle x_{1}, \ldots, x_{n-1}\right\rangle$, then $x_{n} \gamma=\left(x_{n} \gamma\right) \gamma$ is in $\left\langle x_{1} \gamma, \ldots, x_{n-1} \gamma\right\rangle$ so that $\operatorname{Im} \gamma=$ $\left\langle x_{1} \gamma, \ldots, x_{n-1} \gamma\right\rangle$. But $\gamma$ has rank $n-1$ and so we have $\left\{x_{1} \gamma, \ldots, x_{n-1} \gamma\right\}$ is independent, a contradiction. Hence $x_{n} \gamma \notin\left\langle x_{1}, \ldots, x_{n-1}\right\rangle$ and it follows that $\left\{x_{1}, \ldots, x_{n-1}, x_{n} \gamma\right\}$ is independent. Now we define $\varepsilon \in \operatorname{End}(A)$ by putting $x_{1} \varepsilon=x_{n} \gamma,\left(x_{n} \gamma\right) \varepsilon=x_{n} \gamma$ and $x_{i} \varepsilon=x_{i}$ for $2 \leqq i \leqq n-1$. Then $\varepsilon^{2}=\varepsilon$ and $\varepsilon$ has rank $n-1$. We also have $\phi \varepsilon \gamma \in D_{n-1}$ as required.

Corollary 2.4. Every $\mathscr{H}$-class contained in $D_{n-1}$ contains an element which is a product of idempotents.

Proof. Let $H$ be an $\mathscr{H}$-class contained in $D_{n-1}$ and $\alpha$ be a member of $H$. Since $\operatorname{End}(A)$ is regular, there are idempotents $\gamma, \phi$ in $R_{\alpha}$ and $L_{\alpha}$ respectively. By Lemma 2.3, there is an idempotent $\varepsilon$ such that $\gamma \varepsilon \phi \in D_{n-1}$. From the fact that $P_{n-1}$ is completely 0 -simple, it follows that $\gamma \mathscr{R} \gamma \varepsilon \phi \mathscr{L} \phi$ so that $\gamma \varepsilon \phi \in H$.

An immediate consequence of this corollary and Green's Lemmas (see [8, Lemmas II.2.1 and II.2.2]) is the following result.

Corollary 2.5. Let $H$ be a group of $\mathscr{H}$-class in $D_{n-1}$. Then every element in $D_{n-1}$ can be written as a product of elements from $H \cup E_{1}$.

Lemma 2.6. Every element of $D_{n-1}$ is a product of elements of $E_{1}$.

Proof. We use induction on $n$. When $n=1, D_{0}$ is the set of endomorphisms of rank 0 . Either $D_{0}=\varnothing$ and there is nothing to prove or $A$ contains some constants and

$$
D_{0}=\{\alpha \in \operatorname{End}(A): \operatorname{Im} \alpha=\operatorname{Con}\}
$$

In this case, $D_{0}$ consists of idempotents and the result is true.

When $n=2$, let $\{x, y\}$ be a basis for $A$ and consider the $\mathscr{H}$-class

$$
H=\left\{\alpha \in \operatorname{End}(A): \operatorname{Im} \alpha=\langle y\rangle, \operatorname{Ker} \alpha=C g^{A}(x, y)\right\} .
$$

Certainly $H$ is a group $\mathscr{H}$-class because it contains the idempotent $\eta$ given by $x \eta=y$, $y \eta=y$. If $\alpha \in H$, then $x \alpha=y \alpha=a$ for some element $a$ of $\langle y\rangle$. Define $\varepsilon_{1}$ and $\varepsilon_{2}$ in $\operatorname{End}(A)$ by putting $x \varepsilon_{1}=y \varepsilon_{1}=x$ and $x \varepsilon_{2}=a, y \varepsilon_{2}=y$. Then $\alpha=\varepsilon_{1} \varepsilon_{2}$ and $\varepsilon_{1}, \varepsilon_{2}$ are clearly idempotents of rank 1 . Thus every member of $H$ is a product of idempotents (of rank 1 ) and it follows from Corollary 2.5 that the same is therefore true of $D_{1}$.

Now assume that the result holds for $n-1$ where $3 \leqq n$. Let $\left\{x_{1}, \ldots, x_{n}\right\}$ be a basis for $A$ and consider the $\mathscr{H}$-class 


$$
H=\left\{\alpha \in \operatorname{End}(A): \operatorname{Im} \alpha=\left\langle x_{2}, \ldots, x_{n}\right\rangle, \operatorname{Ker} \alpha=\operatorname{Cg}^{A}\left(x_{1}, x_{2}\right)\right\} .
$$

The idempotent $\theta$ is in $H$ where $x_{1} \theta=x_{2} \theta=x_{2}$ and $x_{i} \theta=x_{i}$ for $i=3, \ldots, n$. Thus $H$ is a group $\mathscr{H}$-class. For $\alpha \in H$ we have $x_{1} \alpha=x_{2} \alpha$ and hence

$$
\operatorname{Im} \alpha=\left\langle x_{2} \alpha, \ldots, x_{n} \alpha\right\rangle
$$

and consequently, $\left\{x_{2} \alpha, \ldots, x_{n} \alpha\right\}$ is independent. Since $x_{1} \notin \operatorname{Im} \alpha$ it follows that $\left\{x_{1}, x_{2} \alpha, \ldots, x_{n} \alpha\right\}$ is independent and hence this set is a basis for $A$. We use this basis to define $\psi \in \operatorname{End}(A)$ by putting $x_{1} \psi=x_{2} \alpha$ and $\left(x_{i} \alpha\right) \psi=x_{i} \alpha$ for $i=2, \ldots, n$. Then $\psi$ is an idempotent of rank $n-1$.

We define $\phi$ to be the idempotent endomorphism of rank $n-1$ given by $x_{1} \phi=x_{2} \phi=$ $x_{1}$ and $x_{i} \phi=x_{i}$ for $i=3, \ldots, n$.

Now consider the algebra $B=\left\langle x_{2}, \ldots, x_{n}\right\rangle$ and define the endomorphism $\beta^{\prime}$ of $B$ by specifying

$$
x_{2} \beta^{\prime}=x_{3} \beta^{\prime}=x_{3} \alpha \text { and } x_{i} \beta^{\prime}=x_{i} \alpha \text { for } i=4, \ldots, n .
$$

Then $\operatorname{Im} \beta^{\prime}=\left\langle x_{3} \alpha, \ldots, x_{n} \alpha\right\rangle$ so that $\beta^{\prime}$ has rank $n-2$. By the induction assumption, $\beta^{\prime}=\varepsilon_{1}^{\prime} \ldots \varepsilon_{k}^{\prime}$ for some idempotents of $\operatorname{rank} n-2$ in $\operatorname{End}(B)$. Now define $\varepsilon_{i} \in \operatorname{End}(A)$ for $i=1, \ldots, k$ by putting $x_{1} \varepsilon_{i}=x_{1}$ and $x_{j} \varepsilon_{i}=x_{j} \varepsilon_{i}^{\prime}$ for $j=2, \ldots, n$. Clearly, each $\varepsilon_{i}$ is an idempotent of rank $n-1$. If we put $\beta=\varepsilon_{1} \ldots \varepsilon_{k}$, then it is readily verified that $\alpha=\phi \beta \psi$ so that the members of $H$ are products of idempotents of rank $n-1$. It now follows from Corollary 2.5 that every member of $D_{n-1}$ is a product of idempotent of rank $n-1$ and this completes the proof by induction.

Theorem 2.1 now follows immediately from Lemmas 2.6 and 2.2. We can deduce a stronger result from Theorem 2.1 and Lemma 2.2. Let $E_{n-r}$ be the set of idempotents of End $(A)$ having rank $r$.

Corollary 2.7. If $A$ is an independence algebra of finite rank $n$, then $K(n, r)=\left\langle E_{n-r}\right\rangle$ for $r=1, \ldots, n-1$.

Proof. The case $r=n-1$ is simply a restatement of the theorem and so we may assume that $r<n-1$. In view of Theorem 2.1 every element of the $\mathscr{D}$-class $D_{r}$ is certainly a product of idempotents. Hence by Lemma 1 of [6], any element $\alpha$ of $D$, is a product of idempotents all of which are $\mathscr{D}$-related to $\alpha$, that is, in $D_{r}$. The result now follows from Lemma 2.2 .

Finally, we remark that both Theorem 2.1 and Corollary 2.7 specialise immediately to give the corresponding results for the full transformation semigroup on a finite set, the monoid of endomorphisms of a finite dimensional vector space and the endomorphism monoid of a free $G$-set of finite rank. 


\section{REFERENCES}

1. R. J. H. Dawlings, Semigroups of singular endomorphisms of vector spaces ( $\mathrm{PhD}$ thesis, $\mathrm{St}$ Andrews, 1980).

2. R. J. H. Dawuings, The semigroup of singular endomorphisms of a finite dimensional vector space, in Semigroups (Eds. T. E. Hall, P. R. Jones, G. B. Preston, Academic Press, Sydney, 1980), 121-131.

3. J. A. Erdos, On products of idempotent matrices, Glasgow Math. J. 8 (1967), 118-122.

4. V. A. R. GouLD, Endomorphism monoids of independence algebras, preprint.

5. G. GrÄTZER, Universal algebra (Van Nostrand, Princeton, 1968).

6. T. E. Hall, On regular semigroups, J. Algebra 24 (1973), 1-24.

7. J. M. HowIE, The subsemigroup generated by the idempotents of a full transformation semigroup, J. London Math. Soc. 41 (1966), 707-716.

8. J. M. HowIE, An introduction to semigroup theory (Academic Press, London, 1976).

9. R. N. McKenzie, G. F. McNulty and W. F. TAYlor, Algebra, lattices, varieties, Vol. I (Wadsworth, Monterey, 1983).

10. W. Narkiewicz, Independence in a certain class of abstract algebras, Fund. Math. 50 (1961/62), 333-340.

11. M. A. Reynolds and R. P. Sullivan, Products of idempotent linear transformations, Proc. Roy. Soc. Edinburgh A, 100 (1985), 123-138.

Department of Mathematics

UNIVERSITY OF YORK

Heslington

YORK Y01 5DD 